STRUCTURAL BIOLOGY
ISSN 2059-7983

Received 25 October 2019

Accepted 14 January 2020

₹ Current address: Diamond Light Source, UK.

Keywords: molecular replacement; CCP4i2 pipelines; ARCIMBOLDO; type IV pili.

PDB reference: PilA1, 6t8s

Supporting information: this article has supporting information at journals.iucr.org/d

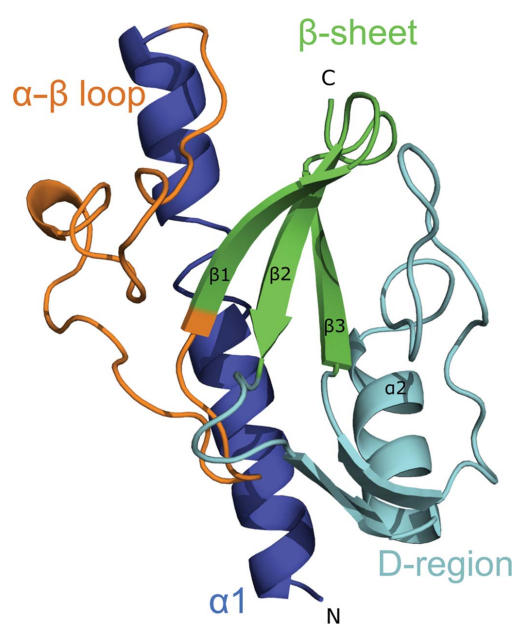

OPEN $\odot$ ACCESS

\section{A practical overview of molecular replacement: Clostridioides difficile PilA1, a difficult case study}

\author{
Adam D. Crawshaw, $¥$ Arnaud Baslé and Paula S. Salgado*
}

Newcastle University Biosciences Institute, Faculty of Medical Sciences, Newcastle University, Newcastle upon Tyne, England. *Correspondence e-mail: paula.salgado@newcastle.ac.uk

Many biologists are now routinely seeking to determine the three-dimensional structures of their proteins of choice, illustrating the importance of this knowledge, but also of the simplification and streamlining of structure-determination processes. Despite the fact that most software packages offer simple pipelines, for the non-expert navigating the outputs and understanding the key aspects can be daunting. Here, the structure determination of the type IV pili (TFP) protein PilA1 from Clostridioides difficile is used to illustrate the different steps involved, the key decision criteria and important considerations when using the most common pipelines and software. Molecular-replacement pipelines within $C C P 4 i 2$ are presented to illustrate the more commonly used processes. Previous knowledge of the biology and structure of TFP pilins, particularly the presence of a long, $\mathrm{N}$-terminal $\alpha$-helix required for pilus formation, allowed informed decisions to be made during the structure-determination strategy. The PilA1 structure was finally successfully determined using ARCIMBOLDO and the $a b$ initio MR strategy used is described.

\section{Introduction}

Once complete diffraction data at an appropriate resolution (usually at least around 3-3.5 $\AA$ ) have been recorded from a protein crystal sample, the next challenge is to solve the phase problem (Taylor, 2003). Phases are required to calculate an electron-density map, from which a model of the structure is built. These are missing from diffraction data experiments and can be experimentally derived using techniques such as single/multiple anomalous dispersion (SAD/MAD) or single/ multiple isomorphous replacement (SIRAS/MIRAS) (Taylor, 2010; Wang et al., 2014). Alternatively, phases can be calculated using similar structures that have already been determined, which is known as molecular replacement (MR). Here, we review an outline of a number of different strategies (Fig. 1) starting with a single sweep of X-ray diffraction data to calculate an electron-density map using molecular replacement. We use the structure determination of the major pilin in type IV pili (TFP), PilA1, from Clostridioides difficile as a working example.

Molecular replacement can be used to determine phases for new structures using diffraction data of reasonable quality $(R$ factors below $50 \%$ ). While high-resolution data are preferable, it is the low-resolution information that is most important for molecular replacement, and there are a number of examples in the PDB of structures phased by molecular replacement where the data do not extend beyond $10 \AA$ resolution (Evans \& McCoy, 2008). MR does not require considerations such as the pre-soaking of crystals with heavy atoms or production, purification and crystallization that 
incorporates an anomalously scattering atom such as selenium, or determining the specific X-ray wavelength at which the data need to be recorded for experimental phasing (Garman \& Murray, 2003). Although techniques such as sulfur SAD (S-SAD) enable experimental phases to be calculated from a 'native crystal', careful design of the diffraction experiment is required to be able to exploit the very weak anomalous differences, in addition to the difficulties in collecting data at the long wavelengths needed (Basu et al., 2019; Bent et al., 2016). Recent advances in both algorithms and instrumentation, including long-wavelength beamlines such as I23 at Diamond Light Source (Aurelius et al., 2017), have rendered S-SAD an increasingly popular structuredetermination method.

In any case, where crystal growth or supply are difficult, being able to determine the structure 'natively' can be particularly advantageous. Molecular replacement is also a powerful way to identify ligands, whether these are potential drugs, substrates or other compounds. After soaking or cocrystallizing experiments using different ligands, data sets recorded from multiple crystals of the same protein species in different conditions are used for phase calculation in search of a positive difference density representing a ligand in a potential binding site (Krojer et al., 2017; Silvestri et al., 2018).

Over the years, a number of different programs have been developed, some of which involve a more hands-on approach requiring suitable search models to be identified and prepared, such as Phaser (McCoy et al., 2007, 2018) and MOLREP (Vagin \& Teplyakov, 2010). Other programs such as AMPLE and $M r B U M P$ provide a more complete pipeline, requiring only scaled reflections, the target sequence and, in the latter case, a small number of fragments provided by another server, Rosetta (Bibby et al., 2012; Rigden et al., 2008). Another approach is to use $B A L B E S$, a fully automated pipeline that uses a reorganized protein structure database derived from the PDB in order to include multimeric and domain organization models (Long et al., 2008). Although these and other MR pipelines are available in different software packages such as CCP4i2 (Potterton et al., 2018) and Phenix (Liebschner et $a l ., 2019)$, here we focus on $C C P 4 i 2$.
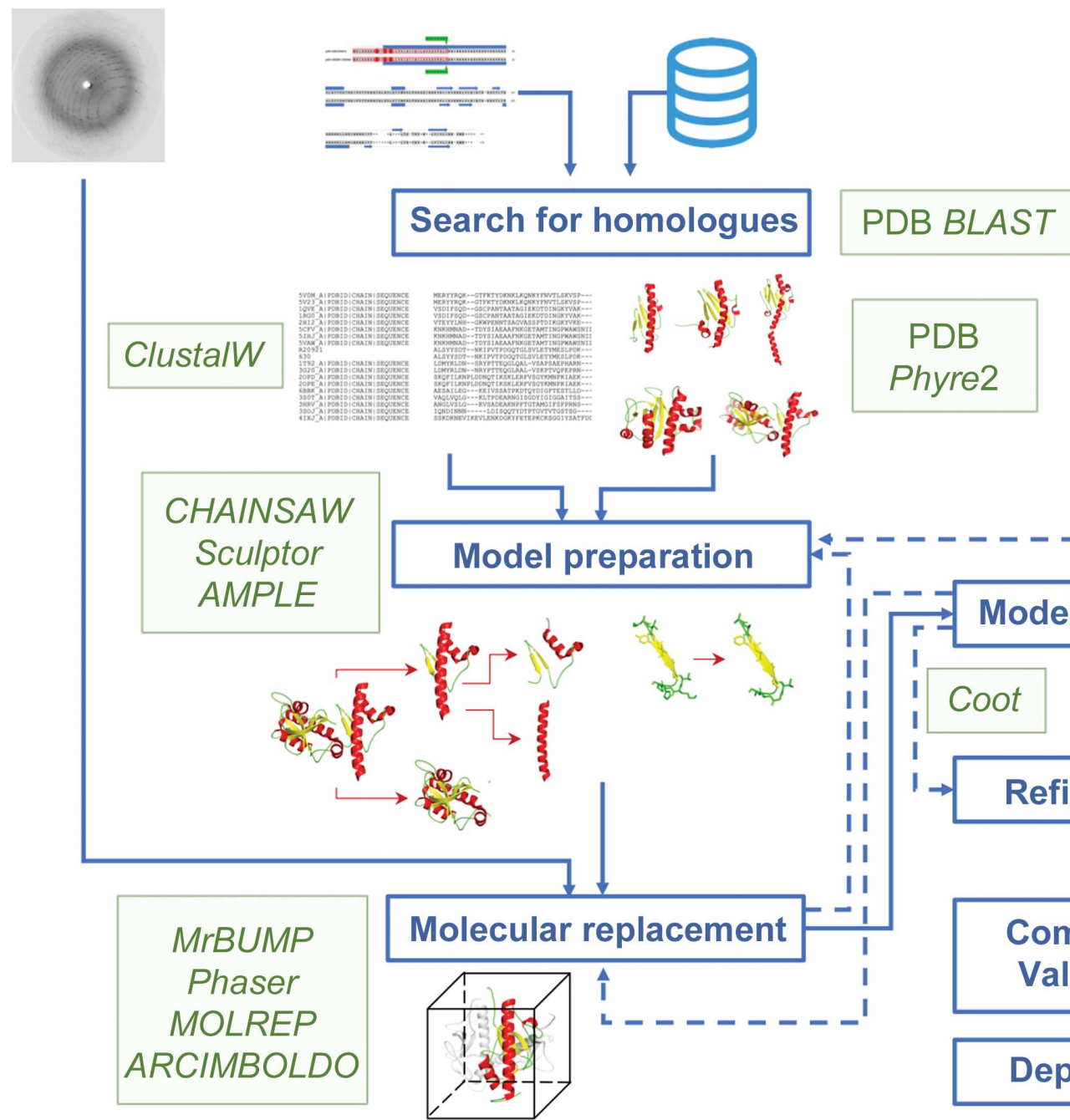

Phyre2 
More recently, it has increasingly become possible to determine phases using methods that do not use structural homologues of the full protein or domains. ARCIMBOLDO uses a fragment-based method and harnesses the predicted secondary structure of the target sequence to place fragments as search models (Millán et al., 2015), in combination with powerful density-modification algorithms such as those implemented in SHELXE (Sheldrick, 2010), to provide an initial trace model (Usón \& Sheldrick, 2018). This ab initio method was initially developed and based on the presence of $\alpha$-helical structures in the target structure and reflections in the X-ray data that extend to approximately $2 \AA$ resolution. More recent versions of $A R C I M B O L D O$ now include the determination of $\beta$-strand structures (Fedosyuk et al., 2016; Sammito et al., 2013) and have also proven successful in solving the phase problem for data to $2.6 \AA$ resolution.

The working example described here is the PilA1 protein from $C$. difficile. PilA1 is the major pilin of the type IV pili (TFP) system in this clinically important Gram-positive bacterium (Maldarelli, Matz et al., 2016). C. difficile is an opportunistic pathogen, particularly affecting individuals with a compromised gut microbiome, often owing to treatment with antibiotics (Huang et al., 2009). Pili are long, proteinaceous filaments which protrude from the surface of bacteria. They are virulence factors which enable host colonization, either by mediating interactions with host tissue, enabling direct binding of the bacteria to the host and/or by mediating inter-bacterial interactions, enabling bacterial aggregation/biofilm formation (Manetti et al., 2007). TFP are the most widespread type of pili, and are uniquely found in both Gram-positive and Gramnegative species (Melville \& Craig, 2013). In C. difficile and related Clostridia, they have been implicated in twitching ability and biofilm formation, both of which are important pathogenicity factors (Maldarelli, Piepenbrink et al., 2016; McKee et al., 2018). C. difficile has two TFP gene clusters, with the primary cluster encoding all of the proteins required for functional pili: the major pilin PilA1; the pre-pilin peptidase PilD; the membrane-associated proteins PilC, PilMN and PilO; the assembly and disassembly ATPases PilB and PilT; and PilU, PilV and PilK, which are known as minor pilins. PilA1 is thought to assemble into long pili filaments, which are decorated by the minor pilins. Pilin proteins can be identified by a highly conserved signal-peptide sequence (Imam et al., 2011) that is cleaved by the pre-pilin peptidase PilD before insertion into the pilin filament (Melville \& Craig, 2013).

There are around 20 protein crystal structures of pilin proteins in the PDB, mostly from Pseudomonas aeruginosa (PDB entry 1qve; Audette et al., 2004), Neisseria gonorrhoeae (PDB entry 2hi2; Craig et al., 2006) and N. meningitis (PDB entry 2opd; Helaine et al., 2007). Pilins exhibit a long $\mathrm{N}$-terminal $\alpha$-helix $(\alpha 1)$ that forms a polymerization stalk domain, and a globular $\mathrm{C}$-terminal domain, described as the headgroup, that consists of an $\alpha-\beta$ region linking the $\alpha 1$ helix to a $\beta$-sheet of at least three strands (Fig. 2; Craig \& Li, 2008). A highly variable region, known as the D-region, is located at the C-terminus of the headgroup; it is predicted to be the most solvent-accessible element and can form interactions with host-cell surfaces (Craig \& $\mathrm{Li}, 2008$ ). The D-region is also delimited by a disulfide bridge in the TFP pilin structures from Gram-negative bacteria determined to date (Craig \& $\mathrm{Li}$, 2008).

Our attempts to determine the structure of a PilA1 construct lacking part of the $\mathrm{N}$-terminal $\alpha 1$ helix to prevent self-polymerization are used here as an example of molecularreplacement approaches.

\section{Methods}

\subsection{Recombinant protein production and purification}

A PilA1 construct from $C$. difficile strain R20291 lacking the first 34 amino acids (PilA1 $11-34$, corresponding to 139 of 173 residues) was donated by Edward Couchman (N. Fairweather group, Imperial College London, England). CD3355 from R20291 genomic DNA was amplified and inserted into an empty pET-28a vector such that it was expressed with a noncleavable $\mathrm{N}$-terminal $6 \times$ His tag. PilA1 $\Delta 1-34$ was expressed in Escherichia coli Rosetta cells by inoculating 11 Luria-Bertani (LB) medium supplemented with $50 \mathrm{mg} \mathrm{ml}^{-1}$ kanamycin and $30 \mathrm{mg} \mathrm{ml}^{-1}$ chloramphenicol with $10 \mathrm{ml}$ of an overnight culture (100 $\mathrm{ml} \mathrm{LB}$ with the same antibiotics). The cells were grown at $37^{\circ} \mathrm{C}$ until an optical density at $600 \mathrm{~nm}$ of $0.4-0.6$ was reached, at which point cultures were induced with isopropyl

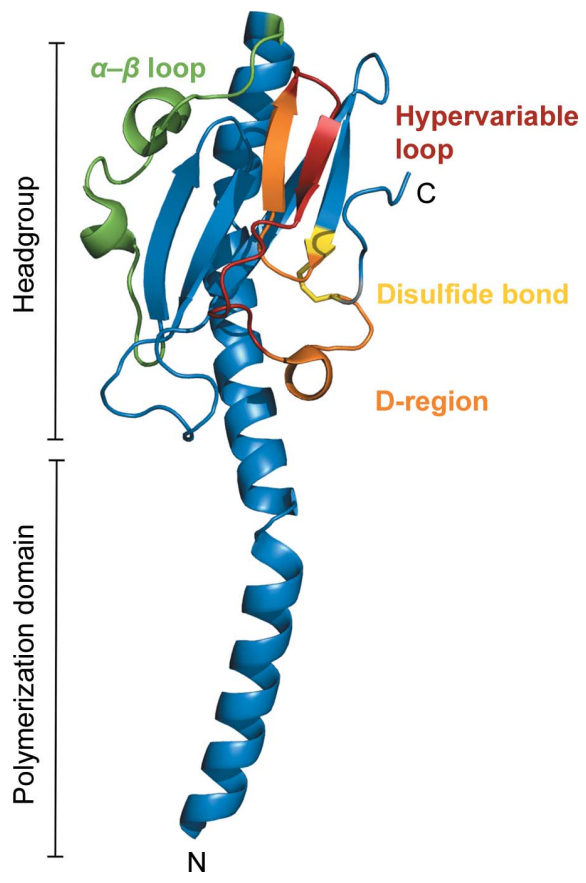

Figure 2

Structural features of TFP pilins. The crystal structure of full-length PilE1 from N. gonorrhoeae (PDB entry 2hi2; Craig et al., 2006) showcasing the distinct domains of TFP pilin proteins: an N-terminal hydrophobic helix $(\alpha 1)$ responsible for pilin polymerization, an $\alpha-\beta$ loop and a D-region. In PilE1 and other TFP pilin structures from Gram-negative bacteria determined to date, the D-region is delimited by a disulfide bond that links the termini of the D-region (Cys121-Cys151 in this case; yellow). This domain exhibits the lowest level of sequence conservation across TFP pilins and contains a hypervariable loop with the highest level of variability. 
Table 1

Data-processing statistics for PilA1.

Values in parentheses are for the outer shell.

\begin{tabular}{ll}
\hline X-ray source & Beamline I04-1, Diamond Light Source \\
Wavelength $(\AA)$ & 0.92 \\
Resolution range $(\AA)$ & $51.21-1.65$ \\
Unit-cell parameters & \\
$\quad a=b, c(\AA)$ & $102.57,104.34$ \\
$\quad \alpha=\beta=\gamma\left({ }^{\circ}\right)$ & 90 \\
Space group & $P 4_{1} 2_{1} 2$ or $P 4_{3} 2_{1} 2$ \\
Total No. of reflections & $964312(39779)$ \\
No. of unique reflections & $67404(3396)$ \\
$\langle I / \sigma(I)\rangle$ & $12.0(0.6)$ \\
$C C_{1 / 2}$ & $0.999(0.390)$ \\
$R_{\text {meas }}$ & $0.142(3.961)[0.05 \dagger]$ \\
Multiplicity & $14.3(11.7)$ \\
Completeness $(\%)$ & $100.0(100.0)$ \\
Mosaicity $\left({ }^{\circ}\right)$ & 0.12 \\
Wilson $B$ factor $\left(\AA^{2}\right)$ & 27.1 \\
\hline
\end{tabular}

$\dagger$ Low-resolution shell.

$\beta$-D-1-thiogalactopyranoside at a final concentration of $1 \mathrm{~m} M$. Protein production was carried out at $20^{\circ} \mathrm{C}$ with agitation at $180 \mathrm{rev} \mathrm{min}^{-1}$ in an incubator for $\sim 20 \mathrm{~h}$. The cells were harvested by centrifugation at $3036 \mathrm{~g}$ for $30 \mathrm{~min}$, resuspended in $20 \mathrm{~m} M$ Tris- $\mathrm{HCl} \mathrm{pH} 8.0,500 \mathrm{mM} \mathrm{NaCl}$, centrifuged at $3011 \mathrm{~g}$ for a further $10 \mathrm{~min}$ and the supernatant was discarded. The cell extracts were purified by nickel-affinity purification on a $5 \mathrm{ml}$ HisTrap column (GE Healthcare) pre-equilibrated with $50 \mathrm{~m} M$ Tris- $\mathrm{HCl}$ pH 8.0, $500 \mathrm{~m} M$ NaCl. His-tagged protein was collected in $2 \mathrm{ml}$ fractions during elution with 50 , $125,250,375$ and $500 \mathrm{mM}$ imidazole in five steps of $15 \mathrm{ml}$. Peak fractions were analyzed by SDS-PAGE, pooled and the volume was reduced to $\sim 2 \mathrm{ml}$ using a $3 \mathrm{kDa}$ molecular-weight cutoff concentrator (Amicon). The sample was loaded onto a Superdex 75 16/600 size-exclusion column (GE Healthcare) pre-equilibrated with $20 \mathrm{~m} M$ Tris- $\mathrm{HCl}$ pH 8.0, $150 \mathrm{mM} \mathrm{NaCl}$ and connected to an ÄKTA pure FPLC system (GE Healthcare). Peak-containing fractions were analyzed by SDS-PAGE and those containing pure protein were pooled and concentrated to $16 \mathrm{mg} \mathrm{ml}^{-1}$ for use in crystallization trials.

\subsection{Crystallization}

Sitting drops of PilA1 $\Delta 1-34$ were dispensed in 1:1 and 2:1 protein:crystallization solution ratios in MRC crystallization plates with a Mosquito dispensing robot (TTP Labtech) using several commercial crystallization screens. Crystals measuring $150 \times 150 \times 100 \mu \mathrm{m}$ were obtained after four days of incubation at $20^{\circ} \mathrm{C}$ in $1.6 \mathrm{M}$ sodium citrate $\mathrm{pH} 6.5$, a condition that provides cryoprotection (Bujacz et al., 2010). Crystals were harvested in nylon loops and directly flash-cooled in liquid nitrogen for subsequent synchrotron data collection (Garman \& Owen, 2006).

\subsection{X-ray diffraction data collection}

Data sets were collected from four independent native crystals on the fixed-wavelength $(\lambda=0.92 \AA)$ beamline I04-1 at Diamond Light Source using a Dectris PILATUS 2M pixelarray detector. Test images of each crystal at $0^{\circ}$ and $90^{\circ}$ were recorded and iMosflm (Battye et al., 2011) was used to index the data and determine the likely Bravais lattice and unit-cell parameters of the crystal. The strategy function of iMosflm was utilized to determine the optimum starting position of the crystal for full, complete native data-set collection. The test images also informed the detector distance settings by visual assessment of the maximum resolution of spots on the test images. The detector distance was set so that the resolution at the edge of the detector was $1.5 \AA$. During the diffraction experiment, the crystal was rotated $200^{\circ}$ with an exposure time of $0.5 \mathrm{~s}$ and each image was recorded over an oscillation of $0.1^{\circ}$. Such a strategy enabled the collection of a native data set with high multiplicity and completeness in order to simplify the structure-solution pipeline (Table 1).

\subsection{X-ray diffraction data processing}

Data were indexed and integrated using DIALS (Gildea et al., 2014), which automatically selected space group $P 4_{1} 2_{1} 2$. It should be noted that this is related to space group $P 4_{3} 2_{1} 2$, its enantiomer, by an inversion at the origin (Shmueli, 2010) and this ambiguity cannot be resolved at this stage. The data were then imported into a $C C P 4$ project using the $C C P 4 i 2 \mathrm{GUI}$ (Potterton et al., 2018) before scaling using AIMLESS (Evans et al., 2011) (Table 1). The resolution cutoff was determined by examining the $\mathrm{CC}_{1 / 2}$ and the $I / \sigma(I)$ in the outer shell to a resolution where the $\mathrm{CC}_{1 / 2}$ was 0.4 (Karplus \& Diederichs, 2015), combined with an $I / \sigma(I)$ of over 0.5 (Evans \& Murshudov, 2013). Assessment of the probabilities of the space-group selection based on systematic absences as calculated by POINTLESS (Evans, 2006), performed as part of the AIMLESS pipeline, did not solve the space-group ambiguity. For both $P 4_{3} 2_{1} 2$ and $P 4_{1} 2_{1} 2$ the probability based on systematic absences was 0.864 , and therefore only solving the phase problem and assessing the resulting electron-density maps will enable the identification of the correct space group. Systematic absences allow discrimination between possibilities within a Laue group, but do not allow the distinction of different enantiomers.

\subsection{Structure determination, model building and validation}

Molecular replacement was carried out within CCP4i2 (Potterton et al., 2018) using Phaser (McCoy et al., 2018) after manual model preparation using Sculptor (Bunkóczi \& Read, 2011) and Coot (Emsley et al., 2010). Ab initio MR with ARCIMBOLDO_LITE (Millán et al., 2015) provided a correct solution, from which a model was built using Buccaneer (Cowtan, 2006). After iterative cycles of model building and refinement with Coot (Emsley et al., 2010) and REFMAC (Murshudov et al., 2011), the model was validated using MolProbity (Williams et al., 2018). Details of these processes are described below and refinement statistics are given in Table 2. 
Table 2

Refinement statistics for PilA1.

Ramachandran statistics and MolProbity clashscore were obtained using MolProbity (Williams et al., 2018)

\begin{tabular}{ll}
\hline Space group & $P 4_{1} 2_{1} 2$ \\
$R_{\text {work }}(\%)$ & 19.4 \\
$R_{\text {free }}(\%)$ & 21.4 \\
No. of non-H atoms & 3069 \\
$\quad$ Protein & 254 \\
$\quad$ Solvent & 400 \\
No. of residues & \\
R.m.s. deviations & 1.30 \\
$\quad$ Bond angles $\left({ }^{\circ}\right)$ & 0.01 \\
$\quad$ Bond lengths $(\AA)$ & \\
Average $B$ factor $\left(\AA^{2}\right)$ & 28.00 \\
$\quad$ Overall & 28.53 \\
Protein & 34.38 \\
$\quad$ Water & \\
Ramachandran plot & 97.21 \\
$\quad$ Most favoured regions $(\%)$ & 2.57 \\
Allowed regions $(\%)$ & 0 \\
$\quad$ Outliers $(\%)$ & 0.48 \\
Clashscore & 0.81 \\
MolProbity clashscore & \\
\hline
\end{tabular}

\section{A manual approach}

Here, we outline a simple manual approach to molecular replacement (Fig. 1) as initially attempted to determine the structure of PilA1.

\subsection{Search for homologues}

Identifying structural models of sequence-related proteins for use as search models is the first step in the molecularreplacement process. Published structures are stored in the Protein Data Bank (PDB), and here we utilize the European instance of the database, PDBe (https://www.ebi.ac.uk/pdbe/; Armstrong et al., 2020). PDBe provides two methods to perform a search of the PDB: by peptide sequence, a simple FASTA sequence search and the more sophisticated PHMMER approach (http://hmmer.org). Other tools such as HHpred (Zimmermann et al., 2018), available as part of the MPI Bioinformatics Toolkit (https://toolkit.tuebingen.mpg.de/), can also be particularly powerful for identifying subunits within a target sequence for which there are known structures.

In these searches, the full-length sequence of PilA1 was used as the search query to ensure the greatest coverage. Using the PHMMER approach at $\mathrm{PDBe}$, a number of structural models were identified with a sequence identity of up to $43 \%$ that could be used as search models. The full-length sequence of PilA1 was also submitted to the HHpred server and a number of fragments for which there are known structures were identified which covered the PilA1 sequence to varying degrees. Additionally, consideration should be made of structures identified during a literature search. While their sequence identity might be low, two proteins may display a greater structural identity and such examples could help to assemble a useful search model. In the case of PilA1, this is particularly stark as pilins, which are also highly related to pseudopilins (Melville \& Craig, 2013), contain common structural motifs including a lengthy $\mathrm{N}$-terminal $\alpha$-helix and a
Table 3

Related homologues used to build the search ensemble and methods of identification.

\begin{tabular}{lll}
\hline & \multicolumn{2}{l}{ PDB Sequence } \\
Protein, organism & code identity (\%) & Method of identification \\
\hline PilX, Neisseria meningitidis & 2opd 38.46 & PDBe BLAST, HHPred \\
Pseudopilin, Escherichia coli & 3g20 28.30 & PDBe BLAST, HHPred \\
PulG, Klebsiella pneumoniae & $1 \mathrm{t} 92 \quad 27.18$ & PDBe BLAST, HHPred \\
PilJ, Clostridioides difficile & 4ixj 19.86 & PDBe BLAST, HHPred \\
PilE, Francisella tularensis & 3soj 14.42 & Literature review \\
PilA, Acinetobacter baumannii & 5vaw 13.49 & Literature review \\
FimU, Pseudomonas aeruginosa & 4ipu 13.51 & HHPred \\
\hline
\end{tabular}

globular region (Fig. 2). Using this knowledge of pilins and their common structural features allowed us to identify other potential search models within known pilin and pseudopilin structures, despite low sequence identity. This is a good example of a situation in which applying prior information on the biological properties of the protein of interest increases the pool of structures available to build a good-quality search model that may not show sequence identity above standard thresholds.

The results from both the PHMMER and HHpred searches were collated and assessed for their suitability. A summary of the models identified and their corresponding sequence identity to PilA1 are summarized in Table 3. A subset of these results with higher similarity were chosen as initial search models; these included PDB entries 4ixj and 2opd. PDB entry 4ixj represents a minor pilin from $C$. difficile (Piepenbrink $e t$ al., 2014), PilJ, which has $20 \%$ identity to the PilA1 sequence. PDB entry 2opd is a pilin from N. meningitidis (Helaine et al., 2007) that displays $38 \%$ sequence identity to the PilA1 sequence.

\subsection{Model preparation}

A selection of tools for molecular-replacement model preparation are available in $C C P 4 i 2$. These include $C C P 4 m g$ and Coot (McNicholas et al., 2011; Emsley et al., 2010) for selecting the components of a search model and directly outputting them to a molecular-replacement process, while CHAINSAW is used for mutating and truncating a search model using sequence alignment with the target sequence (Stein, 2008). In this case, Sculptor was the chosen tool and is outlined in more detail in Bunkóczi \& Read (2011).

The models identified during the search for homologues were downloaded from PDBe in PDB format. They were opened in Coot and chain $A$ of each model was selected and saved in PDB format; this ensured that the file only contained chain $A$ and no additional molecules such as waters or ligands. Within $C C P 4 i 2$, a similar strategy of selecting only protein atoms from a specific chain is also available. The file produced in Coot was then imported into Sculptor in CCP4i2, a tool that can aid the success of molecular replacement by editing the model based on sequence similarity, mutating and/or removing unreliable regions (Bunkóczi \& Read, 2011). To run Sculptor on a model structure, both the search model (search PDB) and a peptide-sequence alignment of the target and search 
proteins must be provided. A suitable alignment can be performed using ClustalW (Larkin et al., 2007), which is available in the CCP4i2 suite as part of the model-preparation tab of the tasks menu. The target and related sequences are inputted in FASTA format and an alignment file (in ALN format) is generated, which is then available within the CCP4i2 Sculptor input form. The search model output by Sculptor is now ready for use in a molecular-replacement process.

To create ensembles containing multiple structures, search models edited to contain a single chain were loaded into Coot and aligned using the secondary-structure matching tool $(S S M)$. The coordinates of the aligned structures were then saved in PDB format. These edited search models were then input into the ensemble builder for Phaser in CCP4i2. The peptide sequences of these models were aligned as described previously and were also added to the input form.

\subsection{Molecular replacement}

Here, we will focus on Phaser (McCoy et al., 2007) as the main tool for MR within $C C P 4 i 2$. The input parameters for basic MR using Phaser require a reflection file, information about the composition of the asymmetric unit and a search model. In $C C P 4 i 2$, the reflection file is selected from the output from AIMLESS. The composition of the asymmetric unit is determined by inputting the sequence of the target protein; a job is then run to calculate the Matthews coefficient, which allows an estimation of the likely number of molecules in the asymmetric unit. In the case of PilA1, the Matthews coefficient was ambiguous between either three or four possible copies in the asymmetric unit. Finally, the search model, provided as a PDB file containing either a single search model or an ensemble of search models, is selected. In this section, Phaser can also be given information regarding the similarity of the search models to the target protein either as a sequence-identity score or a root-mean-squared (r.m.s.) value, indicating the expected deviation between the target and search structures (Supplementary Fig. S1).

At the end of the process, Phaser provides two key scores that must be assessed to determine whether a correct solution with phases that allow the calculation of an interpretable electron-density map has been obtained: the TFZ score and the LLG. A TFZ (translation-function $Z$-score) value above 8 indicates that the solution has definitely been found, while a score of 7 or lower suggests that it is increasingly less likely that a correct solution has been obtained. During the Phaser process, the LLG is also a good indicator of whether the solution is correct, and this value should increase greatly as a correct solution is found (usually to greater than 100). Both of these values are displayed in the Phaser job window in CCP4i2 as the job runs and can be found in the results window once the process has completed (Supplementary Fig. S2). In the case of PilA1 using our ensemble, these values are a TFZ of 5.7 and a final LLG of 29, indicating that this was not a correct solution. When viewed in Coot the map was discontinuous, also confirming that the phase problem had not been solved.
Owing to the space-group ambiguity, the search was carried out in Phaser selecting all possible enantiomorphs. The selected solution was in space group $P 4_{3} 2_{1} 2$, the enantiomorph of $P 4_{1} 2_{1} 2$, but the TFZ scores for other possibilities were similarly low. In any case, this is an ambiguity that would only be clearly resolved if the phase problem had been correctly solved.

Unfortunately, in the case of PilA1, we have not successfully found a solution using this method of MR. However, this is a useful approach to use, especially if more similar structural homologues are available. Fortunately, other methods are available which utilize MR without the need to collect any further diffraction data, such as experimental phasing data.

\subsection{Ab initio MR}

Molecular replacement without search models is becoming ever more possible on a desktop computer, using programs such as AMPLE and ARCIMBOLDO. ARCIMBOLDO uses a fragment-based approach, placing short fragments from secondary-structure predictions to determine the initial phases (Millán et al., 2015). ARCIMBOLDO uses Phaser to place these fragments before density modification using SHELXE (Sheldrick, 2010). PilA1 is a promising candidate for $A R C I M B O L D O$, with the full-length PilA1 predicted to contain an extended $\mathrm{N}$-terminal $\alpha$-helix of approximately 60 residues and the diffraction data collected to a resolution of greater than $2 \AA$.

Tools such as PSIPRED (http://bioinf.cs.ucl.ac.uk/psipred/) and JPred (http://www.compbio.dundee.ac.uk/jpred/) allow secondary-structure predictions when provided with a peptide sequence (Buchan \& Jones, 2019; Jones, 1999; Drozdetskiy et al., 2015). The output of a PSIPRED analysis of the truncated construct of PilA1 used here predicts a 30-residue-long $\alpha$-helix, as expected based on our knowledge of TFP pilins. The ARCIMBOLDO_LITE distribution can now be used on a single multi-core machine and is distributed with $C C P 4 i 2$. The information required to run a job includes scaled reflection data (MTZ format), the molecular mass of the target protein and the number of copies in the asymmetric unit. As the PilA1 crystals are calculated to contain either three or four copies in the asymmetric unit, two separate processes were run searching for three or four copies of an $\alpha$-helix containing 30 residues.

In the case of PilA1, after approximately $20 \mathrm{~h}$ of run time on a modest desktop machine, ARCIMBOLDO_LITE was able to provide a solution. An electron-density map, a PDB file of the backbone of the best solution and an HTML-based report were output (Fig. 3a). In line with the SHELX package, the success of the process is assessed using a correlation coefficient (CC). A correct solution is likely to have been found when the CC is greater than 25\% (Usón \& Sheldrick, 2018). The final CC for the PilA1 process is $42.3 \%$, indicating that a correct solution has been determined (Fig. 1a). Additionally, 378 out of 432 residues were built, including the key structural features of TFP (details in Fig. 2): one N-terminal $\alpha$-helix from each molecule (each of approximately 30 residues), as well as 
the $\beta$-sheet characteristic of the head domain. On inspection of the electron-density map, good continuity is observed, a further indication that the phases had been correctly calculated and a solution had been found (Fig. 3b).

In the case of PilA1, $a b$ initio MR methods were successful in solving the phase problem, rather than using a search model that was based on sequence homology. As such, the output of $A R C I M B O L D O$ was used for model building and refinement. statistics may be high $(\sim 0.5)$ but will decrease with further rounds of refinement, which involves iterative cycles of automated refinement using REFMAC (Murshudov et al., 2011), followed by inspection and manual adjustment in Coot. As the resolution of the PilA1 $\Delta 1-34$ data $(1.65 \AA)$ is borderline for the use of anisotropic $B$ factors in refinement (Merritt, 2012), an isotropic refinement protocol was used throughout. Coot was used to refine local bond angles, check the sequence

\subsection{Model building, refinement} and validation

Two model-building tools are provided in CCP4i2: ARP/wARP (Chojnowski et al., 2019) and Buccaneer (Cowtan, 2006). For the purposes of this workflow, we used Buccaneer to build upon the polyalanine model that was generated by ARCIMBOLDO. Buccaneer has two input modes in $C C P 4 i 2$ that are tailored to phase problems that have been solved using experimental phasing or using molecular replacement. For PilA1, we selected the molecularreplacement mode and therefore we must input the search model that was used for structure determination. In this case, we can input the polyalanine model generated by ARCIMBOLDO_ LITE. We must also input the scaled reflections and the free $R$ set that were generated by AIMLESS. Finally, we must define the crystal contents by inputting the target sequence. These are the only essential pieces of information that Buccaneer requires to run the process, but a large number of more advanced parameters can also be input. For the case of PilA1, the default values were used. After model building, Buccaneer also utilizes REFMAC (Murshudov et al., 2011) to perform an initial round of refinement. As well as visually inspecting the resulting output, it is also important to note the statistics of the completed job, including the number of residues that have been built as well as the refinement measures $R_{\text {work }}$ and $R_{\text {free. }}$. These initial refinement

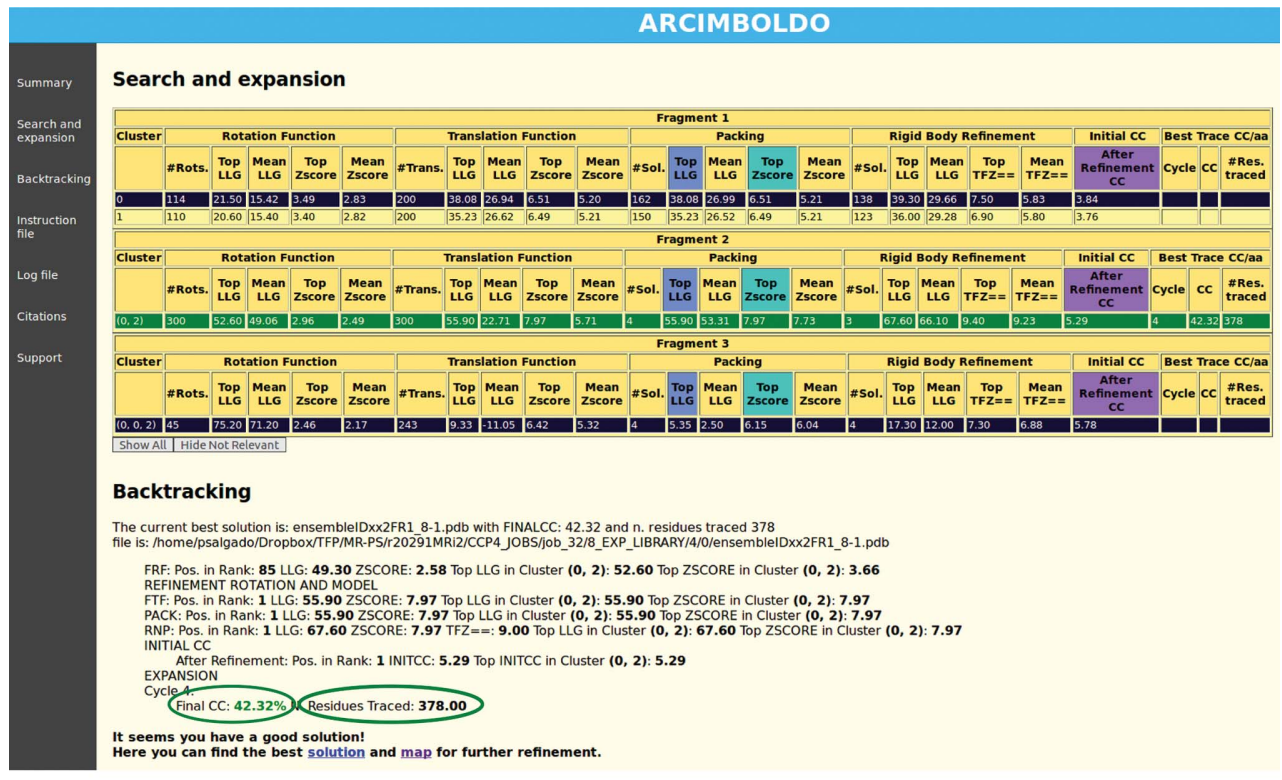

(a)

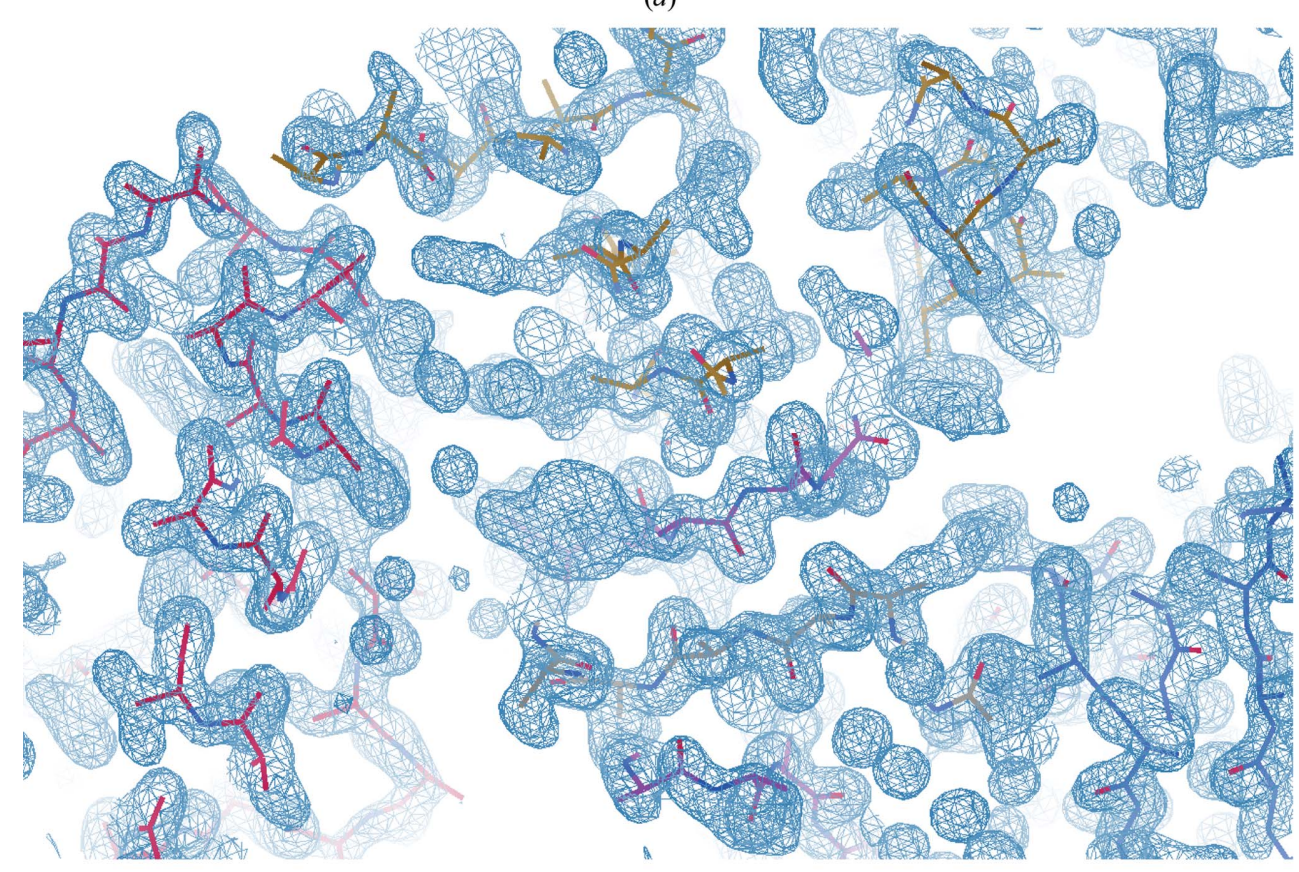

(b)

Figure 3

Ab initio MR with ARCIMBOLDO_LITE. (a) HTML-based ARCIMBOLDO_LITE output, showing that three 30-residue $\alpha$-helical fragments have been found and the corresponding statistics. The overall correlation coefficient (CC) was $43.2 \%$, as highlighted, and 378 residues have been traced. (b) Electrondensity map (blue chicken wire) at a $1.5 \sigma$ contour level showing that well defined density and clearly identifiable side chains were obtained. The traced residues are built as polyalanine chains (stick representation) in $A R C I M B O L D O \_L I T E$, which fitted well into the electron-density map. 
register with the electron density, add alternate side-chain conformations and add water molecules. After several rounds of refinement, $R_{\text {work }}$ and $R_{\text {free }}$ values of $19.4 \%$ and $21.4 \%$, respectively, were achieved (Brünger, 1992, 1993).

Structure validation was performed using MolProbity (Williams et al., 2018). This software also identified and flipped any aspartate, glutamate or histidine residues that clashed and added riding hydrogens so that hydrogen-bonding potential can be assessed (Chen et al., 2010). Clashing side chains, unfavoured rotamers and Ramachandran outliers were identified by MolProbity and problematic residues were inspected and manually fixed using Coot whenever the electron-density maps failed to support the unusual conformations. After a further round of REFMAC refinement and assessment in MolProbity, it was noted that there were still a number of bond lengths and angles with root-mean-squared deviations (r.m.s.d.s) outside the acceptable ranges (Rossmann \& Arnold, 2001). A weighting term was applied in REFMAC, also under restraints in the input form, starting at 0.1 and incremented in steps of 0.5. The output of these was assessed in MolProbity until there were no outliers unless justified by the electron-density maps and the $R_{\text {work }}$ and $R_{\text {free }}$ values were closely observed to ensure that there was no bias occurring from the weighting. A final weighting term of 0.1 was chosen.
Final CIF files were prepared using the $C C P 4 i 2$ interface under the deposition section of the task menu for analysis using the PDBe validation server (https://validate-pdbe. wwpdb.org/). Once validated, the files were uploaded to the PDBe deposition server (https://deposit-pdbe.wwpdb.org/) with PDB identifier 6t8s.

\section{PilA1 structure}

The structure of R20291 PilA1 $\Delta 1-34$ (Fig. 4) is typical of the TFP pilins, containing a long $\mathrm{N}$-terminal $\alpha$-helix $(\alpha 1)$ which is linked by an $\alpha-\beta$ loop to an antiparallel $\beta$-sheet $(\beta 1-\beta 2-\beta 3)$ that encapsulates the D-region containing a shorter $\alpha$-helix $(\alpha 2)$, with the rest of this subdomain largely formed of loop regions. As there are no cysteine residues in PilA1, it lacks the disulfide bridge that delimits the D-region of Gram-negative pilins.

The crystal structures of PilA1 from R20291 and two other strains were published in 2015, and although these structures included a maltose-binding protein (MBP) tag, the structure of the R20291 PilA1 monomer was essentially the same (Piepenbrink et al., 2015). The core r.m.s.d. between our model and the published structure, as calculated using the secondarystructure matching tool in Coot, was $0.89 \AA$. However, Piepenbrink and coworkers determined the structures of MBP-PilA1 fusions, while the structure described here contains a much shorter $6 \times$ His tag, allowing us to observe PilA1 multimers which provide possible PilA1 interfaces. There are three molecules in the asymmetric unit and interactions within this arrangement could be biologically relevant for pilus formation, so the coordinates were submitted to the Protein Interfaces, Surfaces and Assemblies (PISA) server (Krissinel \& Henrick, 2007) for investigation. PISA scores interfaces between molecules using a complexformation significance score (CSS), and is used to assess whether molecular interfaces are biologically significant or a product of crystallization: 0.0 indicates no biological significance and a value of 1.0 indicates significant complex formation (Krissinel \& Henrick, 2007). A summary of the interfaces detected by PISA is presented in Supplementary Table S1. Of particular interest was a salt bridge between Asp62 and Lys96

Figure 4

R20291 PilA1 structure. (a) Chain $A$ of R20291 PilA1 $\Delta 1-34$ (cartoon) contains the structural regions associated with TFP pilin structures: a long $\alpha 1$ helix (dark blue) followed by an $\alpha-\beta$ loop that connects the long $\alpha$-helix and an antiparallel $\beta$-sheet (green). The variable D-region (cyan) contains a shorter $\alpha$-helix of ten residues in length, but is mostly composed of flexible loops. (b) Top view of the three molecules observed in the asymmetric unit of R20291 PilA1 $\Delta 1-34$, showing potential pilin-pilin interfaces. (c) Analysis using PISA indicated a possible interface between R20291 PilA1 1 1-34 molecules via an Asp62Lys96 salt bridge. 
in neighbouring R20291 PilA1 1 1-34 molecules (Fig. 4), part of an interface with a calculated surface area representing $7 \%$ of the solvent-accessible area of the pilin. A number of hydrogen-bonding interactions were also predicted to contribute to this interface, indicating that it could be biologically relevant. Moreover, attempts to mutate either residue to alanine resulted in insoluble or no recombinant protein being produced, suggesting that these residues are important for protein stability. Interestingly, this interaction had not been observed in the published structural models (Piepenbrink et al., 2014, 2015) as the presence of the large MBP tag prevented any direct PilA1-PilA1 interactions. Instead, the authors of these studies suggested that a different interaction would be relevant for TFP filament formation based on the models of filament formation proposed for the TFP filaments from $N$. meningitidis, $N$. gonorrhoeae and Vibrio cholerae (Hartung et al., 2011; Craig \& Li, 2008; Craig et al., 2003, 2006). These models were built using crystallographic and NMR structures of the pilin proteins and electron-microscopy data of the full filament structure to fit the pilin units (Craig \& Li, 2008). Such models place the extended, hydrophobic N-terminal $\alpha$-helix within the core of the filament, interacting with other pilin units via hydrophobic interactions. Based on the similarities between PilA1 and these structures, Piepenbrink et al. (2015) proposed a model for TFP filament formation in $C$. difficile in which a salt bridge between PilA1 units via Lys30 and Glu75 would be equivalent to that observed in the TcpA filament model. However, this salt bridge is not observed in our direct PilA1-PilA1 interactions.

As part of our analysis of the PilA1 structure, we submitted our model to the PDBeFold (Krissinel \& Henrick, 2004) and DALI (Holm \& Rosenström, 2010) servers to identify similar structures in the PDB. This search readily retrieved structures of several TFP pilins, such as the full-length PilE1 (PDB entry 2hi2) from N. gonorrhoeae (Craig et al., 2006) that shared 39\% sequence identity with the $C$. difficile protein. It also retrieved the structures of the major pilins from other Gram-positive bacteria such as TcpA from $V$. cholerae (PDB entries 1oqv and 3hrv; Craig et al., 2003; Lim et al., 2010), CofA from E. coli (PDB entry 3s0t; Kolappan et al., 2012) and PilS from Salmonella typhi (PDB entry 3fhu; Balakrishna et al., 2009). Interestingly, the structures of type II secretion system (TIISS) pseudopilins from Gram-negative bacteria were also retrieved, such as PulG (PDB entry 1t92) from Klebsiella pneumoniae (Köhler et al., 2004), EspG from $V$. cholerae (PDB entry 4lw9; F. S. Vago, K. Raghunathan, J. C. Jens, W. J. Wedemeyer, M. Bagdasarian, J. S. Brunzelle \& D. N. Arvidson, unpublished work) and GspG from E. coli (PDB entry 3g20; Korotkov et al., 2009). Pseudopilins are part of the TIISS, are similar in architecture to TFP and export pilin-like units; however, they do not readily form filaments as observed in TFP (Korotkov et al., 2012). All structures share the commonly observed fold of TFP, including the N-terminal $\alpha$-helix $(\alpha 1)$ connected to the head domain and the variable D-region at the C-termini (Fig. 2). Whether Gram-positive TFP pilins are functionally more similar to the TIISS pseudopilins or the Gram-negative pilins remains to be determined once full- length pilin and filament structures have been determined. It will therefore be important to determine the structures of the full-length PilA1 and other minor pilins, as well as investigate the interactions between these proteins, to elucidate pili filament formation. Together, this information would allow us to start unravelling the mechanisms underlying TFP formation and assembly in C. difficile.

\section{Conclusion}

Despite collecting high-resolution data for R20291 PilA1 $\Delta 1-$ 34 , it was not possible to calculate phases and an interpretable electron-density map using our manually edited search models based on known pilin structures. As the native data set for R20291 PilA1 $\Delta 1-34$ had high resolution $(1.65 \AA)$, high completeness, low $R_{\text {meas }}$ and high $I / \sigma(I)$, it was an ideal candidate for ab initio phasing using ARCIMBOLDO (Millán et al., 2015). As our example shows, using a careful approach to molecular-replacement pipelines and exploring available options, combined with prior knowledge of both the biology and structural characteristics of the protein of interest, can lead to solution of the phase problem and the determination of novel protein structures.

\section{Acknowledgements}

We would like to thank Diamond Light Source for beamtime (proposal mx-13587) and all staff at the MX beamlines for their help and fantastic support. We thank Ed Couchman and Neil Fairweather for supplying the initial PilA1-containing plasmid.

\section{Funding information}

This work was carried out during ADC's PhD studies, part of the Newcastle/Durham/Liverpool BBSRC Doctoral Training Program, with support from Medical Research Council UK New Investigator Research Grant MR/M000923/1 to PSS.

\section{References}

Armstrong, D. R., Berrisford, J. M., Conroy, M. J., Gutmanas, A., Anyango, S., Choudhary, P., Clark, A. R., Dana, J. M., Deshpande, M., Dunlop, R., Gane, P., Gáborová, R., Gupta, D., Haslam, P., Koča, J., Mak, L., Mir, S., Mukhopadhyay, A., Nadzirin, N., Nair, S., Paysan-Lafosse, T., Pravda, L., Sehnal, D., Salih, O., Smart, O., Tolchard, J., Varadi, M., Svobodova-Vařeková, R., Zaki, H., Kleywegt, G. J. \& Velankar, S. (2020). Nucleic Acids Res. 48, D335-D343.

Audette, G. F., Irvin, R. T. \& Hazes, B. (2004). Biochemistry, 43, 11427-11435.

Aurelius, O., Duman, R., El Omari, K., Mykhaylyk, V. \& Wagner, A. (2017). Nucl. Instrum. Methods Phys. Res. B, 411, 12-16.

Balakrishna, A. M., Saxena, A. M., Mok, H. Y. \& Swaminathan, K. (2009). Proteins, 77, 253-261.

Basu, S., Olieric, V., Leonarski, F., Matsugaki, N., Kawano, Y., Takashi, T., Huang, C.-Y., Yamada, Y., Vera, L., Olieric, N., Basquin, J., Wojdyla, J. A., Bunk, O., Diederichs, K., Yamamoto, M. \& Wang, M. (2019). IUCrJ, 6, 373-386.

Battye, T. G. G., Kontogiannis, L., Johnson, O., Powell, H. R. \& Leslie, A. G. W. (2011). Acta Cryst. D67, 271-281. 
Bent, A. F., Mann, G., Houssen, W. E., Mykhaylyk, V., Duman, R., Thomas, L., Jaspars, M., Wagner, A. \& Naismith, J. H. (2016). Acta Cryst. D72, 1174-1180.

Bibby, J., Keegan, R. M., Mayans, O., Winn, M. D. \& Rigden, D. J. (2012). Acta Cryst. D68, 1622-1631.

Brünger, A. T. (1992). Nature, 355, 472-475.

Brünger, A. T. (1993). Acta Cryst. D49, 24-36.

Buchan, D. W. A. \& Jones, D. T. (2019). Nucleic Acids Res. 47, W402W407.

Bujacz, G., Wrzesniewska, B. \& Bujacz, A. (2010). Acta Cryst. D66, 789-796.

Bunkóczi, G. \& Read, R. J. (2011). Acta Cryst. D67, 303-312.

Chen, V. B., Arendall, W. B., Headd, J. J., Keedy, D. A., Immormino, R. M., Kapral, G. J., Murray, L. W., Richardson, J. S. \& Richardson, D. C. (2010). Acta Cryst. D66, 12-21.

Chojnowski, G., Pereira, J. \& Lamzin, V. S. (2019). Acta Cryst. D75, 753-763.

Cowtan, K. (2006). Acta Cryst. D62, 1002-1011.

Craig, L. \& Li, J. (2008). Curr. Opin. Struct. Biol. 18, 267-277.

Craig, L., Taylor, R. K., Pique, M. E., Adair, B. D., Arvai, A. S., Singh, M., Lloyd, S. J., Shin, D. S., Getzoff, E. D., Yeager, M., Forest, K. T. \& Tainer, J. A. (2003). Mol. Cell, 11, 1139-1150.

Craig, L., Volkmann, N., Arvai, A. S., Pique, M. E., Yeager, M., Egelman, E. H. \& Tainer, J. A. (2006). Mol. Cell, 23, 651-662.

Drozdetskiy, A., Cole, C., Procter, J. \& Barton, G. J. (2015). Nucleic Acids Res. 43, W389-W394.

Emsley, P., Lohkamp, B., Scott, W. G. \& Cowtan, K. (2010). Acta Cryst. D66, 486-501.

Evans, G., Axford, D. \& Owen, R. L. (2011). Acta Cryst. D67, 261270.

Evans, P. (2006). Acta Cryst. D62, 72-82.

Evans, P. \& McCoy, A. (2008). Acta Cryst. D64, 1-10.

Evans, P. R. \& Murshudov, G. N. (2013). Acta Cryst. D69, 1204-1214.

Fedosyuk, S., Bezerra, G. A., Radakovics, K., Smith, T. K., Sammito, M., Bobik, N., Round, A., Ten Eyck, L. F., Djinović-Carugo, K., Usón, I. \& Skern, T. (2016). PLoS Pathog. 12, e1006079.

Garman, E. \& Murray, J. W. (2003). Acta Cryst. D59, 1903-1913.

Garman, E. F. \& Owen, R. L. (2006). Acta Cryst. D62, 32-47.

Gildea, R. J., Waterman, D. G., Parkhurst, J. M., Axford, D., Sutton, G., Stuart, D. I., Sauter, N. K., Evans, G. \& Winter, G. (2014). Acta Cryst. D70, 2652-2666.

Hartung, S., Arvai, A. S., Wood, T., Kolappan, S., Shin, D. S., Craig, L. \& Tainer, J. A. (2011). J. Biol. Chem. 286, 44254-44265.

Helaine, S., Dyer, D. H., Nassif, X., Pelicic, V. \& Forest, K. T. (2007). Proc. Natl Acad. Sci. USA, 104, 15888-15893.

Holm, L. \& Rosenström, P. (2010). Nucleic Acids Res. 38, W545W549.

Huang, H., Weintraub, A., Fang, H. \& Nord, C. E. (2009). Int. J. Antimicrob. Agents, 34, 516-522.

Imam, S., Chen, Z., Roos, D. S. \& Pohlschröder, M. (2011). PLoS One, 6, e28919.

Jones, D. T. (1999). J. Mol. Biol. 292, 195-202.

Karplus, P. A. \& Diederichs, K. (2015). Curr. Opin. Struct. Biol. 34, 60-68.

Köhler, R., Schäfer, K., Müller, S., Vignon, G., Diederichs, K., Philippsen, A., Ringler, P., Pugsley, A. P., Engel, A. \& Welte, W. (2004). Mol. Microbiol. 54, 647-664.

Kolappan, S., Roos, J., Yuen, A. S., Pierce, O. M. \& Craig, L. (2012). J. Bacteriol. 194, 2725-2735.

Korotkov, K. V., Sandkvist, M. \& Hol, W. G. J. (2012). Nat. Rev. Microbiol. 10, 336-351.

Korotkov, K. V., Gray, M. D., Kreger, A., Turley, S., Sandkvist, M. \& Hol, W. G. J. (2009). J. Biol. Chem. 284, 25466-25470.

Krissinel, E. \& Henrick, K. (2004). Acta Cryst. D60, 2256-2268.

Krissinel, E. \& Henrick, K. (2007). J. Mol. Biol. 372, 774-797.

Krojer, T., Talon, R., Pearce, N., Collins, P., Douangamath, A., Brandao-Neto, J., Dias, A., Marsden, B. \& von Delft, F. (2017). Acta Cryst. D73, 267-278.
Larkin, M. A., Blackshields, G., Brown, N. P., Chenna, R., McGettigan, P. A., McWilliam, H., Valentin, F., Wallace, I. M., Wilm, A., Lopez, R., Thompson, J. D., Gibson, T. J. \& Higgins, D. G. (2007). Bioinformatics, 23, 2947-2948.

Liebschner, D., Afonine, P. V., Baker, M. L., Bunkóczi, G., Chen, V. B., Croll, T. I., Hintze, B., Hung, L.-W., Jain, S., McCoy, A. J., Moriarty, N. W., Oeffner, R. D., Poon, B. K., Prisant, M. G., Read, R. J., Richardson, J. S., Richardson, D. C., Sammito, M. D., Sobolev, O. V., Stockwell, D. H., Terwilliger, T. C., Urzhumtsev, A. G., Videau, L. L., Williams, C. J. \& Adams, P. D. (2019). Acta Cryst. D75, 861-877.

Lim, M. S., Ng, D., Zong, Z., Arvai, A. S., Taylor, R. K., Tainer, J. A. \& Craig, L. (2010). Mol. Microbiol. 77, 755-770.

Long, F., Vagin, A. A., Young, P. \& Murshudov, G. N. (2008). Acta Cryst. D64, 125-132.

Maldarelli, G. A., Matz, H., Gao, S., Chen, K., Hamza, T., Yfantis, H. G., Feng, H. \& Donnenberg, M. S. (2016). J. Vaccines Vaccin. 7, 321.

Maldarelli, G. A., Piepenbrink, K. H., Scott, A. J., Freiberg, J. A., Song, Y., Achermann, Y., Ernst, R. K., Shirtliff, M. E., Sundberg, E. J., Donnenberg, M. S. \& von Rosenvinge, E. C. (2016). Pathog. Dis. 74, ftw061.

Manetti, A. G. O., Zingaretti, C., Falugi, F., Capo, S., Bombaci, M., Bagnoli, F., Gambellini, G., Bensi, G., Mora, M., Edwards, A. M., Musser, J. M., Graviss, E. A., Telford, J. L., Grandi, G. \& Margarit, I. (2007). Mol. Microbiol. 64, 968-983.

McCoy, A. J., Grosse-Kunstleve, R. W., Adams, P. D., Winn, M. D., Storoni, L. C. \& Read, R. J. (2007). J. Appl. Cryst. 40, 658-674.

McCoy, A. J., Oeffner, R. D., Millán, C., Sammito, M., Usón, I. \& Read, R. J. (2018). Acta Cryst. D74, 279-289.

McKee, R. W., Aleksanyan, N., Garrett, E. M. \& Tamayo, R. (2018). Infect. Immun. 86, e00943-17.

McNicholas, S., Potterton, E., Wilson, K. S. \& Noble, M. E. M. (2011). Acta Cryst. D67, 386-394.

Melville, S. \& Craig, L. (2013). Microbiol. Mol. Biol. Rev. 77, 323341.

Merritt, E. A. (2012). Acta Cryst. D68, 468-477.

Millán, C., Sammito, M. \& Usón, I. (2015). IUCrJ, 2, 95-105.

Murshudov, G. N., Skubák, P., Lebedev, A. A., Pannu, N. S., Steiner, R. A., Nicholls, R. A., Winn, M. D., Long, F. \& Vagin, A. A. (2011). Acta Cryst. D67, 355-367.

Piepenbrink, K. H., Maldarelli, G. A., de la Peña, C. F. M., Mulvey, G. L., Snyder, G. A., De Masi, L., von Rosenvinge, E. C., Günther, S., Armstrong, G. D., Donnenberg, M. S. \& Sundberg, E. J. (2014). J. Biol. Chem. 289, 4334-4345.

Piepenbrink, K. H., Maldarelli, G. A., Martinez de la Peña, C. F., Dingle, T. C., Mulvey, G. L., Lee, A., von Rosenvinge, E., Armstrong, G. D., Donnenberg, M. S. \& Sundberg, E. J. (2015). Structure, 23, 385-396.

Potterton, L., Agirre, J., Ballard, C., Cowtan, K., Dodson, E., Evans, P. R., Jenkins, H. T., Keegan, R., Krissinel, E., Stevenson, K., Lebedev, A., McNicholas, S. J., Nicholls, R. A., Noble, M., Pannu, N. S., Roth, C., Sheldrick, G., Skubak, P., Turkenburg, J., Uski, V., von Delft, F., Waterman, D., Wilson, K., Winn, M. \& Wojdyr, M. (2018). Acta Cryst. D74, 68-84.

Rigden, D. J., Keegan, R. M. \& Winn, M. D. (2008). Acta Cryst. D64, 1288-1291.

Rossmann, M. G. \& Arnold, E. (2001). Editors. International Tables for Crystallography, Vol. F. Dordrecht: Kluwer Academic Publishers.

Sammito, M., Millán, C., Rodríguez, D. D., de Ilarduya, I. M., Meindl, K., De Marino, I., Petrillo, G., Buey, R. M., de Pereda, J. M., Zeth, K., Sheldrick, G. M. \& Usón, I. (2013). Nat. Methods, 10, 10991101.

Sheldrick, G. M. (2010). Acta Cryst. D66, 479-485.

Shmueli, U. (2010). Editor. International Tables for Crystallography, Vol. $B$, 2nd online ed. Chester: IUCr. 
Silvestri, I., Lyu, H., Fata, F., Boumis, G., Miele, A. E., Ardini, M., Ippoliti, R., Bellelli, A., Jadhav, A., Lea, W. A., Simeonov, A., Cheng, Q., Arnér, E. S. J., Thatcher, G. R. J., Petukhov, P. A., Williams, D. L. \& Angelucci, F. (2018). ACS Chem. Biol. 13, 21902202.

Stein, N. (2008). J. Appl. Cryst. 41, 641-643.

Taylor, G. (2003). Acta Cryst. D59, 1881-1890.

Taylor, G. L. (2010). Acta Cryst. D66, 325-338.

Usón, I. \& Sheldrick, G. M. (2018). Acta Cryst. D74, 106-116.

Vagin, A. \& Teplyakov, A. (2010). Acta Cryst. D66, 22-25.
Wang, J., Li, Y. \& Modis, Y. (2014). Acta Cryst. D70, 18731883.

Williams, C. J., Headd, J. J., Moriarty, N. W., Prisant, M. G., Videau, L. L., Deis, L. N., Verma, V., Keedy, D. A., Hintze, B. J., Chen, V. B., Jain, S., Lewis, S. M., Arendall, W. B. III, Snoeyink, J., Adams, P. D., Lovell, S. C., Richardson, J. S. \& Richardson, D. C. (2018). Protein Sci. 27, 293-315.

Zimmermann, L., Stephens, A., Nam, S.-Z., Rau, D., Kübler, J., Lozajic, M., Gabler, F., Söding, J., Lupas, A. N. \& Alva, V. (2018). J. Mol. Biol. 430, 2237-2243. 